PROCEEDINGS OF THE

AMERICAN MATHEMATICAL SOCIETY

Volume 137, Number 5, May 2009, Pages 1711-1721

S 0002-9939(08)09766-9

Article electronically published on December 11, 2008

\title{
ASYMPTOTIC ANALYSIS OF A DIRICHLET PROBLEM FOR THE HEAT EQUATION ON A COATED BODY
}

\author{
JINGYU LI, STEVE ROSENCRANS, XUEFENG WANG, AND KAIJUN ZHANG
}

(Communicated by Walter Craig)

\begin{abstract}
Of concern is the protection from overheating of an isotropically conducting body by an anisotropically conducting coating which is thin compared to the scale of the body. We assume either that the whole thermal tensor of the coating is small or that it is small in the directions normal to the body (a case we call "optimally aligned coating"). We study the asymptotic behavior of the solution to the heat equation with Dirichlet boundary conditions on the outer surface of the coating, as the thickness of the coating shrinks. We obtain the exact scaling relations between the thermal tensor and the thickness of the coating so that the effective (limiting) condition on the boundary of the body is of Dirichlet, Robin or Neumann type, with the last condition indicating good insulation.
\end{abstract}

\section{INTRODUCTION}

In [9], we studied the scenario of protecting an isotropically conducting body from overheating by using an insulating coating which is allowed to be anisotropic (e.g. a space shuttle painted with a nanocomposite insulator). Our interest is in finding the right scaling for the thickness of the coating so that the body will be protected most efficiently.

The mathematical model is as follows. Let the domain $\Omega_{1} \subset \mathbb{R}^{n}$ (representing the isotropic body) be surrounded by another domain $\Omega_{2} \subset \mathbb{R}^{n}$ (representing the anisotropic coating). See Figure 1 . Let $\Omega=\bar{\Omega}_{1} \cup \Omega_{2}$. Assume that the thickness of $\Omega_{2}$ is uniform and is equal to $\delta$. Let the thermal tensor $A(x)$ of $\Omega$ be given by

$$
A(x)=\left(a_{i j}(x)\right)_{n \times n}=\left\{\begin{aligned}
k I_{n \times n}, & x \in \Omega_{1}, \\
\sigma\left(\bar{a}_{i j}(x)\right)_{n \times n}, & x \in \Omega_{2},
\end{aligned}\right.
$$

where $k>0$ is the constant thermal conductivity of $\Omega_{1}, I$ is the identity matrix, $\sigma$ is a small positive parameter, the matrix $\left(\bar{a}_{i j}(x)\right)$ is symmetric and positive definite at every $x \in \Omega_{2}$, with its smallest eigenvalue bounded from below by a positive constant, and each $\bar{a}_{i j}$ is a bounded measurable function on $\Omega_{2}$. $\sigma$ being small means that the thermal conductivity of $\Omega_{2}$ is small in all directions (we shall also consider the case where the thermal conductivity of $\Omega_{2}$ is small only in the direction normal to $\left.\partial \Omega_{1}\right)$. See [1] and 11] for the homogenization theory that gives rise to

Received by the editors May 19, 2008.

2000 Mathematics Subject Classification. Primary 35K05, 35K20, 35R05, 80A20, 80M35.

Key words and phrases. Overheating, thin insulator, Dirichlet problem, heat equation, asymptotic analysis, effective boundary condition. 
such anisotropic thermal tensors; see 12 for explicit formulas for these tensors. Suppose the whole system $\Omega$ is subject to high exterior temperature $H$ (as in the case, for example, of a returning space shuttle). Then the temperature function $Q(x, t)$ is the solution of a Dirichlet boundary value problem for the heat equation:

$$
\begin{cases}Q_{t}-\nabla \cdot(A \nabla Q)=0, & x \in \Omega, t>0, \\ Q=H, & x \in \partial \Omega, t>0, \\ Q=Q_{0}(x), & x \in \Omega, t=0,\end{cases}
$$

where the constant $H$ is large compared to the values of $Q_{0}(x)$.

The eigenfunction expansion of $Q$ is given by the following explicit formula:

$$
Q(x, t)=H+\sum_{m \geq 1} e^{-\lambda_{m} t} \phi_{m}(x) \int_{\Omega} \phi_{m}\left(x^{\prime}\right)\left(Q_{0}\left(x^{\prime}\right)-H\right) d x^{\prime},
$$

where $\left(\lambda_{m}, \phi_{m}\right)$ are the eigenvalues and normalized eigenfunctions of the elliptic operator $u \rightarrow-\nabla \cdot(A \nabla u)$ under Dirichlet boundary conditions on $\partial \Omega$. Recall that $0<\lambda_{1}<\lambda_{2} \leq \lambda_{3} \cdots \rightarrow \infty$ and that $\phi_{1}(x)$ can be taken to be positive in the interior of $\Omega$. In particular, $Q(x, t) \rightarrow H$ as $t \rightarrow \infty$. Thus to protect the body $\Omega_{1}$ from overheating, we need to slow down the rate of convergence of $Q$ to $H$. To achieve this, it was argued in 9 that (a) the first Dirichlet eigenvalue $\lambda_{1}$ should be suppressed; (b) the first eigenfunction $\phi_{1}(x)$ should be made to take large values on $\Omega_{1}$; (c) as many $\left\{\lambda_{m}\right\}$ as possible should be suppressed; (d) the higher eigenfunctions should take small values on $\Omega_{1}$. It was proved in 9 ] that both (a) and (b) occur if the coating is "not too thin" in the sense of

$$
\frac{\sigma}{\delta} \rightarrow 0 \text { as } \delta \rightarrow 0^{+}
$$

and that (a)-(d) all occur if the coating is "optimally thick" in the sense of

$$
\frac{\sigma}{\delta^{2}} \rightarrow 0 \text { as } \delta \rightarrow 0^{+} \text {. }
$$

(Friedman 4] and Panasenko [8] also obtained related results on the first eigenpair.)

In this paper, we study the asymptotic behavior of the solution of the heat equation (1.2) directly, and obtain the following result. Suppose

$$
\lim _{\delta \rightarrow 0^{+}} \frac{\sigma}{\delta} \text { exists and }=\alpha \in[0, \infty] .
$$

Then for any fixed, finite time $T>0, Q \rightarrow v$ in $L^{2}\left(\Omega_{1} \times(0, T)\right)$ as $\delta \rightarrow 0^{+}$, where $v$ is the solution of

$$
\begin{cases}v_{t}-k \Delta v=0, & x \in \Omega_{1}, t>0 \\ \partial v / \partial \nu+\left(\alpha \nu \cdot \nu_{\bar{A}} / k\right)(v-H)=0, & x \in \partial \Omega_{1}, t>0 \\ v=Q_{0}, & x \in \Omega_{1}, t=0\end{cases}
$$

with $\nu=\left(\nu_{1}, \cdots, \nu_{n}\right)$ being the unit outer normal vector field on $\partial \Omega_{1}$ and $\nu_{\bar{A}}$ the conormal $\bar{a}_{i j} \nu_{j}$. If $\alpha=+\infty$, then the boundary condition is understood as the Dirichlet boundary condition $v=H$. See Theorem 2.2.

In other words, if $\alpha=0$, i.e. if (1.4) holds, then on the finite time interval $[0, T]$, $\partial \Omega_{1}$ is effectively well-insulated; otherwise, if $0<\alpha \leq \infty$, then $\partial \Omega_{1}$ is subject to either a high exterior temperature $H$ or a large heat flux $\alpha \nu \cdot \nu_{\bar{A}}(H-v)$. These results confirm the rule: to protect $\Omega_{1}$ from overheating, the coating should be at least as thick as required in (1.4). We emphasize that these results are available for a limited (i.e., fixed, finite) time only. 
These results still hold even if the entire thermal tensor is not small over $\Omega_{2}$ in the sense of (1.1), as long as it is small over $\Omega_{2}$ in the direction normal to the body $\Omega_{1}$. This is the case of what we called "optimally aligned coating" in [9]: for $x \in \Omega_{2}$, let $p$ be the projection of $x$ on $\partial \Omega_{1}$; then the vector $\overline{p x}$ is an eigenvector corresponding to the smallest eigenvalue of the thermal tensor $\left(a_{i j}(x)\right)$. See Theorem 2.3, which says that the magnitude of the other eigenvalues is not significant.

We remark that if the insulator $\Omega_{2}$ is not uniformly thick but is thicker than $\delta$, then we can compare the solution $Q$ of (1.2) with the solution $Q^{\prime}$ of (1.2) with $\Omega_{2}$ replaced by $\Omega_{2}^{\prime}$, where $\Omega_{2}^{\prime}$ is contained in $\Omega_{2}$ and has uniform thickness equal to $\delta$ : By the maximum principle for weak solutions (see Theorem 7.2 of [7]), we have $Q(x, t) \leq H$ for $x \in \bar{\Omega}_{1} \cup \Omega_{2}, t \in(0, T)$; and then from the maximum principle again, we find that $Q(x, t) \leq Q^{\prime}(x, t)$ for $x \in \bar{\Omega}_{1} \cup \Omega_{2}^{\prime}, t \in(0, T)$. Note that $Q^{\prime}$ approaches the solution $v$ of (1.6) as $\delta \rightarrow 0$. Thus, roughly speaking, $Q(x, t)$ is either smaller than or close to $v(x, t)$ for $x \in \Omega_{1}$ and $t \in(0, T)$.

The "interior reinforcement problem" for elliptic and parabolic equations was first studied by Sanchez-Palencia [10]. The elliptic problem corresponding to our parabolic one was studied by Brezis, Caffarelli and Friedman [3] (see also Buttazzo and Kohn 2 for the case of rapidly oscillating thickness of the coating). The method of [3] is based on $H^{2}$ a priori estimates, while that of [2] is based on $\Gamma$-convergence. We prove our results using only the parabolic $W^{1,1}$ a priori estimates; we are unaware of any prior papers (except our [9]) that cover the case of optimally aligned coating. We have obtained parabolic $W^{2,1}$ a priori estimates, but as they are not needed for Theorems 2.2 2.3, we do not present them here. (However, $W^{2,1}$ estimates imply that the $L^{2}$ convergence in Theorems $2.2,2.3$ can be strengthened to convergence in $C^{\frac{1}{2}, \frac{1}{4}}\left(\bar{\Omega}_{1} \times[0, T]\right)$ in the case $n=1$.)

\section{Results AND PROOFS}

Let $u(x, t)=Q(x, t)-H$ and $\varphi(x)=Q_{0}(x)-H$; then (1.2) with a given source term is transformed to

$$
\begin{cases}u_{t}-\nabla \cdot(A \nabla u)=f, & (x, t) \in Q_{T}, \\ u=0, & (x, t) \in S_{T}, \\ u=\varphi(x), & x \in \Omega, t=0,\end{cases}
$$

where $Q_{T}=\Omega \times(0, T), S_{T}=\partial \Omega \times(0, T)$. Denote by $W_{2}^{1,1}\left(Q_{T}\right)$ the Hilbert space

$$
\left\{u: u, \nabla_{x} u, u_{t} \in L^{2}\left(Q_{T}\right)\right\}
$$

equipped with the norm

$$
\|u\|_{W_{2}^{1,1}\left(Q_{T}\right)}^{2}:=\|u\|_{L^{2}\left(Q_{T}\right)}^{2}+\left\|\nabla_{x} u\right\|_{L^{2}\left(Q_{T}\right)}^{2}+\left\|u_{t}\right\|_{L^{2}\left(Q_{T}\right)}^{2}
$$

If $\partial \Omega$ is $C^{1}$ smooth, it is well-known that (i) the set of $C^{\infty}$ smooth functions is dense in $W_{2}^{1,1}\left(Q_{T}\right)$, and (ii) $W_{2}^{1,1}\left(Q_{T}\right)$ is continuously embedded in $C\left([0, T] ; L^{2}(\Omega)\right)$. Denote by $W_{2,0}^{1,1}\left(Q_{T}\right)$ the closure in $W_{2}^{1,1}\left(Q_{T}\right)$ of $C^{\infty}$ smooth functions that vanish near $\overline{S_{T}}$. Then it is easy to prove that $W_{2,0}^{1,1}\left(Q_{T}\right)$ is continuously embedded in $C\left([0, T] ; L^{2}(\Omega)\right)$ without assuming any smoothness of $\partial \Omega$. 
The weak solution of (2.1) is a function $u$ satisfying (i) $u \in W_{2,0}^{1,1}\left(Q_{T}\right)$ and $u(\cdot, t) \rightarrow \varphi$ in $L^{2}(\Omega)$ as $t \rightarrow 0^{+}$; (ii) for any $v \in W_{2,0}^{1,1}\left(Q_{T}\right)$, we have

$$
\int_{0}^{T}\left[\int_{\Omega}\left(u_{t} v+a_{i j}(x) u_{x_{i}} v_{x_{j}}\right) d x\right] d t=\int_{0}^{T} \int_{\Omega} f v d x d t
$$

By using eigenexpansions, we can prove that if $f \in L^{2}\left(Q_{T}\right)$ and $\varphi \in H_{0}^{1}(\Omega)$, then (2.1) has one and only one weak solution $u$ which is also in $C\left([0, T] ; H_{0}^{1}(\Omega)\right)$. The weak solution has the following properties, which we present for the case where (1.1) holds: (a) the "transmission condition" on $\partial \Omega_{1} \times(0, T]$ in the weak sense, i.e.

$$
u_{1}=u_{2}, \quad k \frac{\partial u_{1}}{\partial \nu}=\sigma \frac{\partial u_{2}}{\partial \nu_{\bar{A}}},
$$

where $u_{i}$ is the restriction of $u$ on $\Omega_{i} \times(0, T)$, and the conormal derivative is given by $\frac{\partial u}{\partial \nu_{\bar{A}}}=u_{x_{i}} \bar{a}_{i j} \nu_{j}$; (b) if both $f$ and $\varphi$ are bounded, then so is $u$ (Theorem 7.1 of [7]); (c) if $\partial \Omega$ is $C^{2}$ smooth, $f$ is bounded, and $\varphi$ is Hölder continuous on $\bar{\Omega}$ and vanishes on $\partial \Omega$, then $u$ is also Hölder continuous on $\bar{Q}_{T}$ (see Theorem 10.1 of [7]); (d) if $\bar{a}_{i j} \in C^{1}\left(\bar{\Omega}_{2}\right)$, both $\partial \Omega_{i}(i=1,2)$ are $C^{2}$ smooth, and both $f$ and $f_{t}$ are bounded on $Q_{T}$, then $\nabla_{x} u$ is Hölder continuous in each $\bar{\Omega}_{i} \times(0, T]$ and the "transmission condition" holds on $\partial \Omega_{1} \times(0, T]$ in the classical sense; furthermore, $u_{t}$ is continuous on $\bar{\Omega} \times(0, T]$ and $u_{x_{k} x_{j}}, u_{x_{k} t}$ are in each $L^{2}\left(\Omega_{i} \times(0, T)\right)$ (see Theorem 7 of [6]).

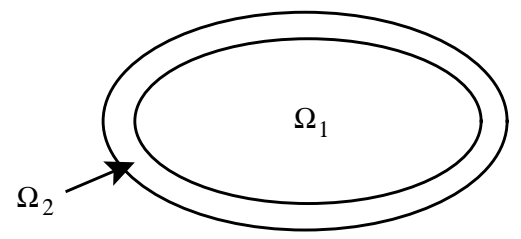

FiguRE $1 . \Omega=\Omega_{2} \cup \bar{\Omega}_{1}, \Omega_{2}$ is an open set, and the distance between $\partial \Omega_{1}$ and $\partial \Omega$ is a positive constant.

For any small positive $\delta$, define $F$ by

$$
\begin{gathered}
F:(p, \tau) \in \partial \Omega_{1} \times[-\delta, \delta] \rightarrow\left(x_{1}, \cdots, x_{n}\right) \in \mathbb{R}^{n}, \quad n>1 \\
\left(x_{1}, \cdots, x_{n}\right)=F(p, \tau)=p+\tau \nu(p) .
\end{gathered}
$$

By Lemma 14.16 of [5], if $\partial \Omega_{1} \in C^{2}$ and $\delta$ is small enough, then $F$ is a diffeomorphism; moreover, if we take

$$
\Omega_{2}=F\left(\partial \Omega_{1} \times(0, \delta)\right),
$$

then $\partial \Omega_{2} \in C^{2}$. For the sake of simplicity, we assume $\partial \Omega_{1}$ is connected.

We need the following a priori estimates. 
Lemma 2.1. Suppose that (1.1) holds, $\partial \Omega_{1} \in C^{2}, f \in L^{2}\left(Q_{T}\right)$ and $\varphi \in H_{0}^{1}(\Omega)$. Then the weak solution $u$ of (2.1) satisfies

$$
\begin{gathered}
\max _{0 \leq t \leq T} \int_{\Omega} u^{2}(x, t) d x \leq C\left(\int_{\Omega} \varphi^{2}(x) d x+\iint_{Q_{T}} f^{2}(x, t) d x d t\right), \\
\iint_{Q_{T}}\left|u_{t}\right|^{2} d x d t+\max _{0 \leq t \leq T}\left[k \int_{\Omega_{1}}|\nabla u|^{2} d x+\sigma \int_{\Omega_{2}}|\nabla u|^{2} d x\right] \\
\leq C\left(\int_{\Omega}\left(\varphi^{2}(x)+|\nabla \varphi|^{2}(x)\right) d x+\iint_{Q_{T}} f^{2}(x, t) d x d t\right),
\end{gathered}
$$

where $C$ is independent of $\sigma$ and $\delta$.

Proof. Estimates (2.5) and (2.6) are part of the standard $L^{2}$ parabolic theory.

Theorem 2.2. Suppose that all the conditions in Lemma 2.1 hold, with

$$
\iint_{\Omega_{2} \times(0, T)} f^{2}(x, t) d x d t
$$

and

$$
\int_{\Omega_{2}}\left(\varphi^{2}+|\nabla \varphi|^{2}\right) d x
$$

being bounded by a constant independent of $\Omega_{2}$ ( $f$ and $\varphi$ are fixed on $\Omega_{1}$ but on $\Omega_{2}$ they are allowed to vary with $\delta$ ), and assume that $\bar{a}_{i j} \in C^{1}\left(\bar{\Omega}_{2}\right)\left(\bar{a}_{i j}\right.$ does not vary with respect to $\delta$ ) and that $\sigma$ remains bounded and

$$
\frac{\sigma}{\delta} \rightarrow \alpha \text { as } \delta \rightarrow 0^{+}
$$

where $\alpha \in[0,+\infty]$. Then the weak solution of (2.1) satisfies $u(x, t) \rightarrow w(x, t)$ strongly in $L^{2}\left(\Omega_{1} \times(0, T)\right)$ as $\delta \rightarrow 0^{+}$, where $w(x, t)$ is the solution of

$$
\begin{cases}w_{t}-k \Delta w=f, & x \in \Omega_{1}, t>0, \\ \partial w / \partial \nu+\alpha \nu \cdot \nu_{\bar{A}} w / k=0, & x \in \partial \Omega_{1}, t>0, \\ w=\varphi(x), & x \in \Omega_{1}, t=0 .\end{cases}
$$

If $\alpha=+\infty$, then the boundary condition is understood as the Dirichlet boundary condition.

Proof. It follows from Lemma 2.1 that for all small $\delta>0$,

$$
\|u\|_{W_{2}^{1,1}\left(\Omega_{1} \times(0, T)\right)} \leq C .
$$

Thus there exists $w \in W_{2}^{1,1}\left(\Omega_{1} \times(0, T)\right)$ such that $u(x, t) \rightarrow w(x, t)$ weakly in $W_{2}^{1,1}\left(\Omega_{1} \times(0, T)\right)$ and strongly in $L^{2}\left(\Omega_{1} \times(0, T)\right)$, after passing to a subsequence of $\delta \rightarrow 0$. Recall that $W_{2}^{1,1}\left(\Omega_{1} \times(0, T)\right)$ is continuously imbedded in $C\left([0, T], L^{2}\left(\Omega_{1}\right)\right)$; thus $u$ and $w$ belong to the latter space. By Lemma $2.1,\|u(\cdot, t)\|_{H^{1}\left(\Omega_{1}\right)}$ is bounded for all small $\delta$, uniformly for $t \in[0, T]$. So by the compact embedding theorem, at each fixed $t, u(\cdot, t) \rightarrow w(\cdot, t)$ after passing to a subsequence of $\delta \rightarrow 0^{+}$. On the other hand, since $\left\|u_{t}\right\|_{L^{2}\left(\Omega_{1} \times(0, T)\right)}$ is bounded, $u(\cdot, t)$ is equicontinuous on $[0, T]$ with respect to the $L^{2}\left(\Omega_{1}\right)$ norm. Now by the Arzela-Ascoli theorem, $u \rightarrow w$ strongly in $C\left([0, T], L^{2}\left(\Omega_{1}\right)\right)$ after passing to a subsequence of $\delta \rightarrow 0^{+}$. Hence the initial condition in (2.7) is satisfied by $w$ in the sense of $w(\cdot, t) \rightarrow \varphi$ in $L^{2}\left(\Omega_{1}\right)$ as $t \rightarrow 0^{+}$.

By the trace theorem (see Lemma 3.4 of [7]) and (2.8), we have

$$
\left\|u_{1}\right\|_{W_{2}^{\frac{1}{2}, \frac{1}{4}}\left(\partial \Omega_{1} \times(0, T)\right)} \leq C \text {. }
$$


So $u_{1} \rightarrow w$ strongly in $L^{2}\left(\partial \Omega_{1} \times(0, T)\right)$ after passing to a subsequence of $\delta \rightarrow 0$.

We shall eventually prove that $w$ is a weak solution of (2.7). Since the weak solution of (2.7) is unique, all the convergence statements made above hold without passing to a subsequence of $\delta \rightarrow 0$.

For any point $p \in \partial \Omega_{1}$, let $l$ be the ray in the conormal direction $\nu_{\bar{A}}$ initiated at $p$. It intersects $\partial \Omega$ at a point $q^{\delta}$. Denote by $q$ the projection of $q^{\delta}$ on $\partial \Omega_{1}$ so that $q^{\delta}=q+\delta \nu(q)$ (see Figure 2). Denote by $h(p)$ the distance between $p$ and $q^{\delta}$.

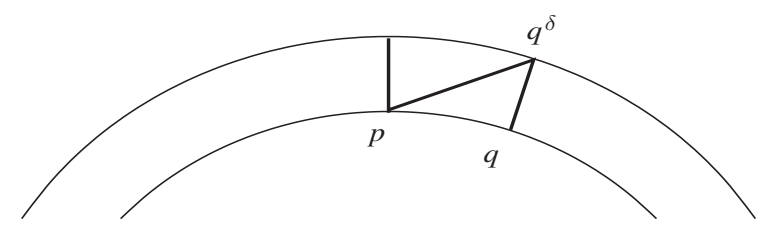

FiguRe 2

Claim. $\frac{h(p)}{\delta} \rightarrow \frac{\left|\nu_{\bar{A}}(p)\right|}{\nu(p) \cdot \nu_{\bar{A}}(p)}$ uniformly for $p$ on $\partial \Omega_{1}$ as $\delta \rightarrow 0$. Let $\beta=h(p) /\left|\nu_{\bar{A}}\right|$. Then

$$
\beta \nu_{\bar{A}}(p)=q-p+\delta \nu(q) .
$$

Now taking the dot-product of (2.10) with $\nu(p)$, we deduce

$$
\beta \nu_{\bar{A}}(p) \cdot \nu(p)=(q-p) \cdot \nu(p)+\delta \nu(q) \cdot \nu(p) .
$$

Recall that $\tau \in C^{2}, \tau \equiv 0$ on $\partial \Omega_{1}$, and $\nabla \tau(p)=\nu(p)$. So Taylor's theorem yields

$$
(q-p) \cdot \nu(p)=O\left(|q-p|^{2}\right) .
$$

Combining this with (2.11), we have $\beta=O\left(|q-p|^{2}+\delta\right)$. Now going back to (2.10), we see that $|q-p|=O\left(|q-p|^{2}+\delta\right)$ and hence $|q-p|=O(\delta)$. This and (2.11) lead to

$$
\beta \nu_{\bar{A}}(p) \cdot \nu(p)=O\left(\delta^{2}\right)+\delta \nu(q) \cdot \nu(p)
$$

from which the claim follows.

Next we need a convenient coordinate system. Reparameterize $\Omega_{2}$ by

$$
\left(x_{1}, \cdots, x_{n}\right)=\bar{F}(p, s)=p+s \frac{\nu_{\bar{A}}}{\left|\nu_{\bar{A}}\right|}(p),
$$

with $s \in(0, h(p))$. For any $q \in \partial \Omega_{1}$, the portion of $\partial \Omega_{1}$ near $q$ can be parameterized as

$$
x_{i}=p_{i}(r), r=\left(r_{1}, \cdots, r_{n-1}\right), 1 \leq i \leq n,
$$

with $p(0)=q$, and with $\frac{\partial p}{\partial r_{i}}(0)$ and $\nu(q)$ forming an orthonormal basis for $\mathbb{R}^{n}$. Write

$$
\frac{\partial\left(p_{1}, \cdots, p_{n}\right)}{\partial\left(r_{1}, \cdots, r_{n-1}\right)}:=\left(\begin{array}{ccc}
\frac{\partial p_{1}}{\partial r_{1}} & \cdots & \frac{\partial p_{n}}{\partial r_{1}} \\
\vdots & \ddots & \vdots \\
\frac{\partial p_{1}}{\partial r_{n-1}} & \cdots & \frac{\partial p_{n}}{\partial r_{n-1}}
\end{array}\right) .
$$


Then the surface element on $\partial \Omega_{1}$ at $q$ is

$$
\begin{aligned}
d S_{p} & =\left.\left(\operatorname{det} \frac{\partial\left(p_{1}, \cdots, p_{n}\right)}{\partial\left(r_{1}, \cdots, r_{n-1}\right)}\left(\frac{\partial\left(p_{1}, \cdots, p_{n}\right)}{\partial\left(r_{1}, \cdots, r_{n-1}\right)}\right)^{\top}\right)^{1 / 2}\right|_{r=0} d r_{1} \cdots d r_{n-1} \\
& =d r_{1} \cdots d r_{n-1}
\end{aligned}
$$

where "T" means transpose. Also, the volume element on $\Omega_{2}$ at $\bar{F}(q, s)$ is

$$
d x_{1} \cdots d x_{n}=\left|\operatorname{det} \frac{\partial \bar{F}}{\partial\left(r_{1}, \cdots, r_{n-1}, s\right)}\right|_{r=0} d r_{1} \cdots d r_{n-1} d s
$$

where

$$
\frac{\partial \bar{F}}{\partial\left(r_{1}, \cdots, r_{n-1}, s\right)}=\left(\begin{array}{ccc}
\frac{\partial p_{1}}{\partial r_{1}}+s \frac{\partial}{\partial r_{1}}\left(\frac{\nu_{\bar{A}_{1}}}{\left|\nu_{\bar{A}}\right|}\right) & \cdots & \frac{\partial p_{n}}{\partial r_{1}}+s \frac{\partial}{\partial r_{1}}\left(\frac{\nu_{\bar{A}_{n}}}{\left|\nu_{\bar{A}}\right|}\right) \\
\vdots & \ddots & \vdots \\
\frac{\partial p_{1}}{\partial r_{n-1}}+s \frac{\partial}{\partial r_{n-1}}\left(\frac{\nu_{\overline{A_{1}}}}{\left|\nu_{\bar{A}}\right|}\right) & \cdots & \frac{\partial p_{n}}{\partial r_{n-1}}+s \frac{\partial}{\partial r_{n-1}}\left(\frac{\nu_{\bar{A}_{n}}}{\left|\nu_{\bar{A}}\right|}\right) \\
\frac{\nu_{\bar{A}_{1}}}{\left|\nu_{\bar{A}}\right|} & \cdots & \frac{\nu_{\bar{A}_{n}}}{\left|\nu_{\bar{A}}\right|}
\end{array}\right)
$$

Let

$$
G(r)=\left(\begin{array}{ccc}
\frac{\partial p_{1}}{\partial r_{1}} & \cdots & \frac{\partial p_{n}}{\partial r_{1}} \\
\vdots & \ddots & \vdots \\
\frac{\partial p_{1}}{\partial r_{n-1}} & \cdots & \frac{\partial p_{n}}{\partial r_{n-1}} \\
\frac{\nu_{\bar{A}_{1}}}{\left|\nu_{\bar{A}}\right|} & \cdots & \frac{\nu_{\bar{A}_{n}}}{\left|\nu_{\bar{A}}\right|}
\end{array}\right)
$$

Since $\partial \Omega_{1} \in C^{2}$ and $\bar{a}_{i j} \in C^{1}\left(\bar{\Omega}_{2}\right)$, we have

$$
\left|\operatorname{det} \frac{\partial \bar{F}}{\partial\left(r_{1}, \cdots, r_{n-1}, s\right)}\right|=|\operatorname{det} G(r)|+O(1) s .
$$

On the other hand, by using the orthonormality of the vectors $\frac{\partial p}{\partial r_{i}}(0)$ and $\nu(q)$, we can easily compute

$$
\operatorname{det} G(0)=\frac{\nu(q) \cdot \nu_{\bar{A}}(q)}{\left|\nu_{\bar{A}}(q)\right|} .
$$

This, together with (2.13), (2.14) and (2.15), implies that the volume element on $\Omega_{2}$ at $\bar{F}(p, s)$ is

$$
d x_{1} \cdots d x_{n}=\left(\frac{\nu \cdot \nu_{\bar{A}}}{\left|\nu_{\bar{A}}\right|}+O(1) s\right) d S_{p} d s
$$

We take

$$
\phi(x):=\left\{\begin{array}{rr}
1-\frac{\tau}{\delta}, & 0 \leq \tau \leq \delta, p \in \partial \Omega_{1}, \\
1, & \text { otherwise }
\end{array}\right.
$$

where we have used the coordinates introduced in (2.4). Observe that $\nabla \phi(x)=$ $\frac{\partial \phi}{\partial \tau} \nu(p)=-\frac{\nu(p)}{\delta}$. For any $\zeta(x, t) \in C^{1}\left(\bar{\Omega}_{1} \times[0, T]\right)$, extend $\zeta(x, t)$ along $\nu_{\bar{A}}$ to $\Omega$ 
by $\zeta(p, s, t)=\zeta(p, t)$ for every $p \in \partial \Omega_{1}$; then it is easy to verify that $\zeta(x, t) \in$ $W_{2}^{1,1}(\Omega \times(0, T))$. By (2.2), we have

$$
\begin{aligned}
& \int_{0}^{T}\left[\int_{\Omega_{1}} u_{t} \zeta d x+\int_{\Omega_{2}} u_{t} \phi \zeta d x+k \int_{\Omega_{1}} \nabla u \cdot \nabla \zeta d x\right. \\
& \left.\quad+\sigma \int_{\Omega_{2}} \bar{a}_{i j}(x) u_{x_{i}}(\phi \zeta)_{x_{j}} d x\right] d t=\int_{0}^{T}\left[\int_{\Omega_{1}} f \zeta d x+\int_{\Omega_{2}} f \phi \zeta d x\right] d t .
\end{aligned}
$$

By using Hölder's inequality and Lemma 2.1, we have

$$
\begin{gathered}
\left|\int_{0}^{T} \int_{\Omega_{2}} f \phi \zeta\right| \leq\left(\int_{0}^{T} \int_{\Omega_{2}} f^{2}\right)^{1 / 2}\left(\int_{0}^{T} \int_{\Omega_{2}} \zeta^{2}\right)^{1 / 2}=o(1), \\
\left|\int_{0}^{T} \int_{\Omega_{2}} u_{t} \phi \zeta\right| \leq\left(\int_{0}^{T} \int_{\Omega_{2}} u_{t}^{2}\right)^{1 / 2}\left(\int_{0}^{T} \int_{\Omega_{2}} \zeta^{2}\right)^{1 / 2}=o(1), \\
\left|\sigma \int_{0}^{T} \int_{\Omega_{2}} \bar{a}_{i j}(x) u_{x_{i}} \zeta_{x_{j}} \phi\right| \\
\leq \sigma^{1 / 2}\left(\sigma \int_{0}^{T} \int_{\Omega_{2}} \bar{a}_{i j}(x) u_{x_{i}} u_{x_{j}}\right)^{1 / 2}\left(\int_{0}^{T} \int_{\Omega_{2}} \bar{a}_{i j}(x) \zeta_{x_{i}} \zeta_{x_{j}}\right)^{1 / 2}=o(1) .
\end{gathered}
$$

On the other hand,

$$
\begin{aligned}
\sigma & \int_{0}^{T} \int_{\Omega_{2}} \bar{a}_{i j} u_{x_{i}} \phi_{x_{j}} \zeta d x d t \\
= & -\frac{\sigma}{\delta} \int_{0}^{T} \int_{\Omega_{2}} \bar{a}_{i j} u_{x_{i}} \nu_{j} \zeta d x d t \\
= & -\frac{\sigma}{\delta} \int_{0}^{T} \int_{\partial \Omega_{1}} \int_{0}^{h(p)}\left[\bar{a}_{i j}(p, s)-\bar{a}_{i j}(p, 0)\right] u_{x_{i}} \nu_{j} \zeta\left(\frac{\nu \cdot \nu_{\bar{A}}}{\left|\nu_{\bar{A}}\right|}+O(s)\right) d s d S_{p} d t \\
& -\frac{\sigma}{\delta} \int_{0}^{T} \int_{\partial \Omega_{1}} \int_{0}^{h(p)} \bar{a}_{i j}(p, 0) u_{x_{i}} \nu_{j} \zeta\left(\frac{\nu \cdot \nu_{\bar{A}}}{\left|\nu_{\bar{A}}\right|}+O(s)\right) d s d S_{p} d t .
\end{aligned}
$$

Observe that $\left|\bar{a}_{i j}(p, s)-\bar{a}_{i j}(p, 0)\right| \leq C s, \bar{a}_{i j}(p, 0) \nu_{j}=\nu_{\bar{A}}, \zeta(p, s, t)=\zeta(p, 0, t)$, and

$$
u_{2}(p, t)=-\int_{0}^{h(p)} \frac{1}{\left|\nu_{\bar{A}}\right|} \cdot \frac{\partial u_{2}}{\partial \nu_{\bar{A}}} d s
$$

for $t \in[0, T]$. Observe also that

$$
\begin{aligned}
\frac{\sigma}{\delta} \int_{0}^{T} \int_{\partial \Omega_{1}} \int_{0}^{h(p)} s\left|\zeta \nabla u_{2}\right| d s d S_{p} d t & \leq C \sigma\left(\int_{0}^{T} \int_{\Omega_{2}}|\nabla u|^{2}\right)^{\frac{1}{2}}\left(\int_{0}^{T} \int_{\Omega_{2}}|\zeta|^{2}\right)^{\frac{1}{2}} \\
& =o(1),
\end{aligned}
$$

where we have used the claim.

Therefore it follows from (2.16) that

$$
\begin{aligned}
\int_{0}^{T} & {\left[\int_{\Omega_{1}} u_{t} \zeta d x+k \int_{\Omega_{1}} \nabla u \cdot \nabla \zeta d x+\frac{\sigma}{\delta} \int_{\partial \Omega_{1}} \nu \cdot \nu_{\bar{A}} u(p, t) \zeta(p, t) d S_{p}\right] d t } \\
& =\int_{0}^{T} \int_{\Omega_{1}} f \zeta d x d t+o(1) .
\end{aligned}
$$


By this and the fact that $u_{1} \rightarrow w$ weakly in $W_{2}^{1,1}\left(\Omega_{1} \times(0, T)\right)$ and strongly in $L^{2}\left(\partial \Omega_{1} \times(0, T)\right)$, we deduce that

$$
\int_{0}^{T}\left[\int_{\Omega_{1}} w_{t} \zeta d x+k \int_{\Omega_{1}} \nabla w \cdot \nabla \zeta d x+\int_{\partial \Omega_{1}} \alpha \nu \cdot \nu_{\bar{A}} w \zeta d S_{p}\right] d t=\int_{0}^{T} \int_{\Omega_{1}} f \zeta d x d t .
$$

This completes the proof.

We say the coating $\Omega_{2}$ is optimally aligned if at every $x \in \Omega_{2}$, the vector $\overline{p x}$ is an eigenvector of the thermal tensor $\left(a_{i j}(x)\right)$ corresponding to the smallest eigenvalue $\sigma_{\min }(x)$, where $p$ is the projection of $x$ onto $\partial \Omega_{1}$, i.e., $x=p+\tau \nu(p)(\tau>0)$. An example is the $2 \mathrm{D}$ disk of radius $1-\delta$ coated by an annulus of thickness $\delta$, the annulus having thermal tensor

$$
A(x)=\left(\begin{array}{lc}
\sigma x^{2}+y^{2} & x y(-1+\sigma) \\
x y(-1+\sigma) & x^{2}+\sigma y^{2}
\end{array}\right) .
$$

The eigenvalues are $x^{2}+y^{2}$ and $\sigma\left(x^{2}+y^{2}\right)$ with eigenvectors angular and radial respectively.

Theorem 2.3. Assume that $\partial \Omega_{1} \in C^{2}$, and let $f$ and $\varphi$ be as given in Theorem 2.2. Suppose that the coating $\Omega_{2}$ is optimally aligned, that

$$
\sigma_{\min }(x)=\sigma \gamma(x)
$$

where $\sigma$ is a small parameter and $\gamma(x)$ is a positive Lipschitz continuous function in $\bar{\Omega}_{2}$ ( $\gamma$ does not vary with $\left.\delta\right)$, and that $\sigma$ remains bounded and

$$
\frac{\sigma}{\delta} \rightarrow \alpha \text { as } \delta \rightarrow 0^{+}
$$

where $\alpha \in[0,+\infty]$. Then the solution $u \rightarrow w$ as $\delta \rightarrow 0^{+}$strongly in $L^{2}\left(\Omega_{1} \times(0, T)\right)$, where $w(x, t)$ is the solution of

$$
\begin{cases}w_{t}-k \Delta w=f, & x \in \Omega_{1}, t>0 \\ \partial w / \partial \nu+\alpha \gamma w / k=0, & x \in \partial \Omega_{1}, t>0 \\ w=\varphi(x), & x \in \Omega_{1}, t=0\end{cases}
$$

If $\alpha=+\infty$, then the boundary condition is the Dirichlet condition $w=0$.

Proof. The proof is a slight modification of that of Theorem 2.2 without reparameterizing $\Omega_{2}$. First note that Lemma 2.1 still holds. From (2.4), it is clear that the volume element on $\Omega_{2}$ at $F(p, \tau)$ is

$$
d x_{1} \cdots d x_{n}=(1+O(1) \tau) d S_{p} d \tau .
$$

For any $\zeta \in C^{1}\left(\bar{\Omega}_{1} \times[0, T]\right)$, extend $\zeta$ along $\nu(p)$ by $\zeta(p, \tau, t)=\zeta(p, t)$. Using the same test function $\phi \zeta$ and observing that $\nabla \phi(x)=\frac{\partial \phi}{\partial \tau} \nu(p)=-\frac{\nu(p)}{\delta}$ is an eigenvector 
of $a_{i j}(x)$ corresponding to $\sigma_{\min }(x)$, we infer that

$$
\begin{aligned}
& \int_{0}^{T} \int_{\Omega_{2}} a_{i j} u_{x_{i}} \phi_{x_{j}} \zeta d x d t \\
&=-\frac{\sigma}{\delta} \int_{0}^{T} \int_{\Omega_{2}} \gamma(x) \zeta \frac{\partial u}{\partial \nu} d x d t \\
&=-\frac{\sigma}{\delta} \int_{0}^{T} \int_{\partial \Omega_{1}} \int_{0}^{\delta} \gamma(p, \tau) \zeta(p, \tau, t) \frac{\partial u}{\partial \tau}(1+O(\tau)) d \tau d S_{p} d t \\
&=-\frac{\sigma}{\delta} \int_{0}^{T} \int_{\partial \Omega_{1}} \int_{0}^{\delta}[\gamma(p, \tau)-\gamma(p, 0)] \zeta(p, \tau, t) \frac{\partial u}{\partial \tau}(1+O(\tau)) d \tau d S_{p} d t \\
&-\frac{\sigma}{\delta} \int_{0}^{T} \int_{\partial \Omega_{1}} \int_{0}^{\delta} \gamma(p, 0) \zeta(p, \tau, t) \frac{\partial u}{\partial \tau}(1+O(\tau)) d \tau d S_{p} d t .
\end{aligned}
$$

Noticing that $\gamma(p, \tau)-\gamma(p, 0)=O(\tau), \zeta(p, \tau, t)=\zeta(p, 0, t)$ and $u(p, t)=-\int_{0}^{\delta} \frac{\partial u_{2}}{\partial \tau} d \tau$, we have

$$
\int_{0}^{T} \int_{\Omega_{2}} a_{i j} u_{x_{i}} \phi_{x_{j}} \zeta d x d t=\frac{\sigma}{\delta} \int_{0}^{T} \int_{\partial \Omega_{1}} \zeta(p, 0, t) \gamma(p, 0) u(p, 0, t) d S_{p} d t+o(1) .
$$

The other modifications are obvious.

\section{ACKNOWLEDGEMENTS}

The third author is grateful for the hospitality of Northeast Normal University, where part of his work was done. The authors gratefully acknowledge partial support by the Chinese NSF (Grant No. 10571102), the Key Research Project on Science and Technology of the Ministry of Education of China (Grant No. 104072), and the American NSF (Grant DMS-0707796).

\section{REFERENCES}

1. A. Bensoussan, J.-L. Lions and G. Papanicolaou, Asymptotic Analysis for Periodic Structures, North-Holland, Amsterdam, New York, 1978. MR0503330 (82h:35001)

2. G. Buttazzo and R. Kohn, Reinforcement by a thin layer with oscillating thickness, Applied Math. Optimization, 16 (1987), 247-261. MR0901816 (89a:73048)

3. H. Brézis, L. A. Caffarelli and A. Friedman, Reinforcement problems for elliptic equations and variational inequalities, Ann. Mat. Pura Appl., 123 (1980), 219-246. MR0581931(81m:35040)

4. A. Friedman, Reinforcement of the principal eigenvalue of an elliptic operator, Arch. Rational Mech. Anal., 73 (1980), 1-17. MR0555579 (81c:35097)

5. D. Gilbarg and N. S. Trudinger, Elliptic Partial Differential Equations of Second Order, Third Edition, Springer-Verlag, Berlin, 1998. MR1814364 (2001k:35004)

6. O. A. Ladyženskaja, J. Rivkind and N. N. Ural'ceva, The classical solvability of diffraction problems, Proc. Steklov Inst. Math., 92 (1966), 132-166.

7. O. A. Ladyženskaja, V. A. Solonnikov and N. N. Ural'ceva, Linear and Quasilinear Equations of Parabolic Type, Translations of Mathematical Monographs 23, Amer. Math. Soc., Providence, Rhode Island, 1967. MR0241822 (39:3159b)

8. G. P. Panasenko, Asymptotics of the solutions and eigenvalues of elliptic equations with strongly varying coefficients, Soviet Math. Dokl., 21 (1980), 942-947.

9. S. Rosencrans and X. Wang, Suppression of the Dirichlet eigenvalues of a coated body, SIAM J. Appl. Math., 66 (2006), 1895-1916; Corrigendum, SIAM J. Appl. Math., 68 (2008), 1202. MR 2262957 (2007h:35038)

10. E. Sanchez-Palencia, Problèmes de perturbations liés aux phénomènes de conduction à travers des couches minces de grande résistivité, J. Math. Pures Appl., 53 (1974), 251-269. MR0364917 (51:1171) 
11. L. Tartar, An Introduction to the Homogenization Method in Optimal Design, Lecture Notes in Mathematics 1740, Springer-Verlag, Berlin, 2000. MR.1804685

12. X. Zheng, M. G. Forest, R. Lipton, R. Zhou and Q. Wang, Exact scaling laws for electrical conductivity properties of nematic polymer nano-composite monodomains, Adv. Funct. Mat., 15 (2005), 627-638.

School of Mathematics and Statistics, Northeast Normal University, Changchun 130024, People's Republic of China

E-mail address: lijy645@yahoo.com.cn

Department of Mathematics, Tulane University, New Orleans, Louisiana 70118

E-mail address: srosenc@tulane.edu

Department of Mathematics, Tulane University, New Orleans, Louisiana 70118

E-mail address: xdw@math.tulane.edu

School of Mathematics and Statistics, Northeast Normal University, Changchun 130024, People's Republic of China

E-mail address: zhangkj201@nenu.edu.cn 